\title{
Der Einflufs der Temperatur auf die Hydrolyse der Wismuthaloide.
}

\author{
Von \\ W. Henz und Alfired Bulia.
}

In unserer Mitteilung „Über die Hydrolyse der Wismuthaloide“ 1 haben wir Versuche bei höherer Temperatur als $25^{\circ}$, wo wir früher

1 Z. anorg. Chem. 61, 387. - Nachdem in dem am 27. Februar 1909 ausgegebenen Hefte dieser Zeitschrift unsere eben zitierte Mitteilung erschienen war, veröffentlichte Herr Rene Dubrisay in dem Sitzungsberichte der Académie des Sciences vom 29. März (Compt. rend. 148, 830) eine kurze Abhandlung über die hydrolytische Spaltung des Wismutchlorids. Wir möchten nicht unterlassen, hier unsere Priorität zu betonen, da Herr Dubrisay uns nicht zitiert, unsere Arbeit ihm also unbekannt geblieben zu sein scheint. Hervorheben möchten wir auch, dals sich unsere Versuche auf alle Wismuthaloide beziehen, dafs wir die Gleichgewichte nach den verschiedensten Methoden und bis zu den höchsten Konzentrationen hergestellt haben, dafs wir das Massenwirkungsgesetz auf diese Fälle angewendet und den Einflufs von Salzen auf die Hydrolyse des Wismutchlorids studiert haben, was alles seitens des Herrn Dubrisar nicht geschehen ist. Dafs wir die Hydrolyse bei verschiedenen Temperaturen zu untersuchen beabsichtigten, haben wir in unserer Mitteilung Seite 395 angegeben. So weit ein Vergleich unserer Werte mit den Zahlen des Herrn Dunarsay möglich ist - die Versuchsreihen beziehen sich nicht auf die gleiche Temperatur -, stimmen die Versuche leidlich überein, wie die folgende Zusammenstellung lehrt, in der wir unsere bei $25^{\circ}$ angestellten, früher veröffentlichten Versuche nach Art der bei $18^{\circ}$ ausgeführten Bestimmungen des Herrn Dubrisay umgerechnet haben (Grammatome pro Liter).
Herz und Bulla (250)
0.016
0.043
0.246 Wismut
0.634
0.818
1.829 Chlor
Dubrisat $\left(18^{0}\right)$
0.016
0.039
0.237 Wismut
0.594
0.729
1.656 Chlor

Nur der höchste und niedrigste Wert des Herrn Dubrisar stimmen nicht recht in die Reihe unserer Zahlen. Wil halten unsere Analysenmethode, wo Wismut und Säure in getrennten Portionen bestimmt werden, für genauer, als die Arbeitsart des Herrn Dubrisay, der beide Bestimmungen in derselben Lösung nacheinander ausführt. 
arbeiteten, in Aussicht gestellt. Wir wählten diesmal $50^{\circ}$, und es gelang uns unschwer, einen Wasserthermostaten bei dieser Temperatur konstant einzustellen.

Zuerst haben wir durch Analysen nachgewiesen, dafs bei $50^{\circ}$ nur derselbe Bodenkörper wie bei $25^{\circ}$ auftritt, nämlich $\mathrm{BiOCl}$ und $\mathrm{BiOBr}$, so dals ohne Rücksicht auf die Temperatur die Umsetzungsgleichungen lauten:

$\mathrm{BiCl}_{3}+\mathrm{H}_{2} \mathrm{O}=\mathrm{BiOCl}+2 \mathrm{HCl}$ und $\mathrm{BiBr}_{3}+\mathrm{H}_{2} \mathrm{O}=\mathrm{BiOBr}+2 \mathrm{HBr}$.

\section{Die Hydrolyse des Wismutchlorids bei $50^{\circ}$.}

Um die Gleichgewichte herzustellen, brachten wir bestimmte Wismutchloridquantitäten mit wechselnden Wassermengen zusammen, verschlossen die Flaschen mit Glasstöpseln und Gummimembran, schüttelten bis zur Einstellung des Endzustandes (24 Stunden lang) und analysierten das Wismut und die freie Säure in derselben Weise, wie wir es in unserer anfangs zitierten Arbeit angegeben haben. Bei der Wiederholung zeigten sich aber besonders in den Säureergebnissen nicht unerhebliche Fehler, was wir auf Verflüchtigung von Chlorwasserstoff aus den oft sehr konzentrierten Lösungen bei $50^{\circ}$ zurückführen. ${ }^{1}$ Nach rerschiedenen Versuchen, diese Störung zu vermindern, ergab sich uns schliefslich als bestes Mittel, die Schüttelflaschen in Messinghülsen mit federndem Boden und fest aufschraubbarem Deckel einzuschliefsen und dann erst die Erreichung des Gleichgewichtes durch Schütteln zu bewirken. Dadurch wurde der Säureverlust sehr gering gehalten.

Die Resultate unserer Versuche waren folgende:

I. $1 \mathrm{~g} \mathrm{BiCl}_{3}$ auf $60 \mathrm{ccm}$ Wasser: In $10 \mathrm{ccm}$ der klaren Gleichgewichtslösung sind keine Millimol $\mathrm{Bi} \cdots$ und $1.08^{2}$ Millimol $\mathrm{H}$ :

II. $1 \mathrm{~g} \mathrm{BiCl}_{3}$ auf $4 \mathrm{ccm}$ Wasser: In $10 \mathrm{ccm}$ der klaren Gleichgewichtslösung sind 2.69 Millimol Bi* und 10.60 Millimol $\mathrm{H}$ :

III. $1 \mathrm{~g} \mathrm{BiCl}_{3}$ auf $2 \mathrm{ccm}$ Wasser: In $10 \mathrm{ccm}$ der klaren Gleichgewichtslösung sind 7.74 Millimol $\mathrm{Bi}^{\cdots}$ und 15.92 Millimol $\mathrm{H}$ :

IV. $1 \mathrm{~g} \mathrm{BiCl}_{3}$ auf $1 \mathrm{ccm}$ Wasser: In $10 \mathrm{ccm}$ der klaren Gleichgewichtslösung sind 15.92 Millimol $\mathrm{Bi}^{\cdots}$ und $24.66 \mathrm{Millimol} \mathrm{H}$.

$\nabla .1 \mathrm{~g} \mathrm{BiCl}{ }_{3}$ auf $0.33 \mathrm{ccm}$ Wasser: In $10 \mathrm{ccm}$ der klaren Gleichgewichtslösung sind 46.2 Millimol Bi”' und 34.90 Millimol $\mathrm{H}$ :

1 In viel geringerem Mafse ist übrigens dieser Fehler auch schon bei $25^{\circ}$ vorhanden.

2 Das heifst etwa so viel, wie bei der vollständigen Zerlegung von $1 \mathrm{~g}$ $\mathrm{BiCl}_{3}$ durch $60 \mathrm{cem}$ Wasser pro $10 \mathrm{ccm}$ entstehen können. 
VI. $1 \mathrm{~g} \mathrm{BiCl}_{3}$ auf $0.25 \mathrm{ccm}$ Wasser: In $10 \mathrm{ccm}$ der klaren Gleichgewichtslösung sind 55.3 Millimol Bi”' und 37.80 Millimol H:

Bei der Vergleichung dieser Zahlen ergibt sich, dals die Hydrolyse mit steigendem Wassergehalt steigt, und dals die gelösten Wismutmengen durchgehend gröfser, die Säuremengen aber entsprechend kleiner als bei den analogen Versuchen ron $25^{\circ}$ sind. Die Hydrolyse geht also mit steigender Temperatur zurück.

Der anfünglich geschriebenen Umsetzungsgleichung entspricht der Ausdruck des Massenwirkungsgesetzes:

$$
\frac{\left.[\mathrm{BiCl}]_{3}\right]}{[\mathrm{HCl}]^{2}}=\text { konst. }^{1}
$$

Für die obigen Versuche ergibt die Ausrechnung:

$\begin{array}{ccc}\text { Nr. des Versuches } & \text { Quotient } & \text { Konstante } \\ \text { II } & \frac{2.69}{10.60^{2}} & 0.025 \\ \text { III } & \frac{7.74}{15.92^{2}} & 0.031 \\ \text { IV } & \frac{15.92}{24.66^{2}} & 0.026 \\ \text { V } & \frac{46.2}{34.90^{2}} & 0.038 \\ \text { VI } & \frac{55.3}{37.80^{2}} & 0.039\end{array}$

Die Konstanten zeigen - ebenso wie bei $25^{\circ}$ - einen bei den höchsten Konzentrationen steigenden Gang. Über die Erklärung dieses Anwachsens gilt das in unserer vorhergehenden Arbeit Gesagte.

Nachdem wir die Gleichgewichtskonstanten bei $25^{\circ}$ und bei $50^{\circ}$ kennen gelernt haben, liegt es nahe, diese Erfahrungen zu benutzen, um nach der bekannten VAN'T HOFF schen Gleichung:

$$
\frac{d \ln K}{d T}=-\frac{q}{R T^{2}}
$$

die Wärmetönung dieser Reaktion zu berechnen. Wir haben diese Rechnung aber nicht ausgeführt, da die von uns gefundenen Gleichgewichtskonstanten zu wenig bestimmt sind. Dagegen lälst sich ohne weiteres sagen, dafs der von uns mit steigender Temperatur beobachtete Rückgang der Hydrolyse in Übereinstimmung mit der Tatsache steht, dafs nach J. THOMSEN ${ }^{2}$ durch die Zersetzung eines Mols Wismutchlorid 7830 cal. abgegeben werden.

1 Z. anorg. Chem. 61, 391.

2 Ber. deutsch. chem. Ges. 16, 39. 
Die Hydrolyse des Wismutbromids bei $50^{\circ}$.

Die zur Feststellung der Hydrolysengleichgewichte dienenden Versuche wurden genau so wie beim Chlorid ausgeführt.

I. $1 \mathrm{~g} \mathrm{BiBr}_{3}$ auf $60 \mathrm{ccm}$ Wasser: In $10 \mathrm{~cm}$ der klaren Gleichgewichtslösung sind keine Millimol Bi” und $0.785^{1}$ Millimol $\mathrm{H}$.

II. $1 \mathrm{~g} \mathrm{BiBr}_{3}$ auf $10 \mathrm{ccm}$ Wasser: In $10 \mathrm{ccm}$ der klaren Gleichgewichtslösung sind 0.64 Millimol $\mathrm{Bi} \cdots$ und 3.56 Millimol $\mathrm{H}$ :

III. $1 \mathrm{~g} \mathrm{BiBr}_{3}$ auf $4 \mathrm{ccm}$ Wasser: In $10 \mathrm{ccm}$ der klaren Gleichgewichtslösung sind 2.74 Millimol $\mathrm{Bi} \cdots$ and 6.12 Millimol $\mathrm{H}$ :

IV. $1 \mathrm{~g} \mathrm{BiBr}$ auf $2 \mathrm{ccm}$ Wasser: In $10 \mathrm{ccm}$ der klaren Gleichgewichtslösung sind 6.44 Millimol Bi” und 9.62 Millimol $\mathrm{H}$.

V. $1 \mathrm{~g} \mathrm{BiBr}_{3}$ auf $1 \mathrm{ccm}$ Wasser: In $10 \mathrm{ccm}$ der klaren Gleichgewichtslösung sind 12.90 Millimol $\mathrm{Bi}^{\cdots}$ und 15.20 Millimol $\mathrm{H}$.

Beim Vergleich dieser Zahlen mit den entsprechenden Werten bei $25^{\circ}$ zeigt sich, dals nur ganz geringe Differenzen vorhanden sind. Das Hydrolysengleichgewicht ist also von der Temperatur so gut wie unabhängig; demgemäls mufs die Wärmetönung der Wismutbromidhydrolyse klein sein, was ein von uns angestellter - roher Versuch vollkommen bestätigte. Da die Werte von Wismut und Säure im Gleichgewicht etwa ebenso sind wie bei $25^{\circ}$, so können auch die Konstanten des Massenwirkungsgesetzes keine irgend erhebliche Veränderung erfahren haben.

\section{Die Hydrolyse des Wismutjodids bei $50^{\circ}$.}

Ebenso wie bei $25^{\circ}$ erfolgt auch bei $50^{\circ}$ die Zersetzung des Wismutjodids durch Wasser so langsam, dals wir die Gleichgewichtsuntersuchungen nicht weiterführten. Auch ein Versuch, Wismutjodid aus jodwasserstoffsaurer Lösung durch Wasser bei $50^{\circ}$ zu fällen, gab kein Resultat.

Da eine Temperaturveränderung nur einen geringen Einflufs auf diese Hydrolysengleichgewichte hat, bei höherer Temperatur die Fehlerquellen aber beträchtlich wachsen, so werden wir uns bei diesen Ergebnissen bescheiden.

1 Das heifst etwa so viel, wie unter diesen Bedingungen bei der vollständigen Zersetzung des Salzes entstehen können.

Breslau, Chemisches Institut der Universität, 27. April 1909.

Bei der Redaktion eingegangen am 28. April 1909. 